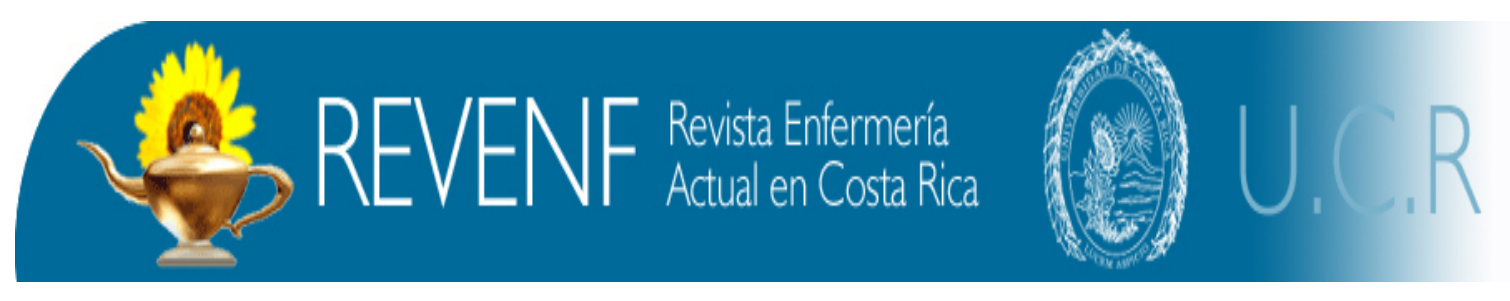

Revista Semestral Número8 Año 4, Abril - Set.2005

ISSN1409 - 4568

\title{
GRUPO TERAPEUTICO DE APOYO DIRIGIDO A MUJERES SOBREVIVIENTES DE VIOLENCIA INTRAFAMILIAR ${ }^{1}$
}

\author{
Elena Mora-Escalante ${ }^{2}$
}

\begin{abstract}
RESUMEN
Uno de los efectos devastadores que tiene la violencia contra las mujeres es la desesperanza aprendida, ya que llegan a creer que no tienen ningún control sobre su situación de violencia y que no pueden hacer nada para cambiarlo.

Considerando lo anterior, fue de mucha importancia desarrollar un grupo terapéutico de apoyo dirigido hacia las necesidades de las mujeres sobrevivientes de VIF.

Para su desarrollo se utilizó la perspectiva fenomenológica, el enfoque género sensitivo, el enfoque humanístico, además de la metodología cualitativa.

El proceso grupal se orientó a explorar los ejes centrales de las áreas afectadas por la VIF, a expresar sentimientos, a producir cambios cognoscitivos y conductuales, logrando el protagonismo de las miembras del grupo.

Los enfoques utilizados en la terapia grupal de apoyo, hizo que se prestara gran atención a las conductas, las ideas y las expresiones de cada mujer, lo que estimuló al grupo para que fueran las mismas participantes las que reconocieran las herramientas para enfrentar la situación de violencia que viven.
\end{abstract}

Palabras claves : Violencia intrafamiliar, enfermería en Salud Mental, Grupo Terapéutico.

${ }^{1}$ Fecha de recepción del original: Noviembre $2004 \quad$ Fecha de aceptación del original: Febrero 2005

${ }^{2}$ Enfermera, licenciada en Salud Mental y Psiquiatría, Profesora Escuela de Enfermería Universidad de Costa Rica, San José, Costa Rica. moresca@costarricense.cr 


\section{ABSTRACT \\ GROUP THERAPY SUPPORT FOR WOMEN WHO SURVIVE INTRA- FAMILIAL VIOLENCE}

The specific violence against women is a reflection of the inequality and inferiority constructed against them in a society of power against one another. One of the terrible effects of the continuous violence and abuse is that these women learn to live without hope.

Because of the above, it was very important to develop of support groups answering the needs of these survivors of intra-familial violence. The development of this therapy used a phenomenological perspective and Sensitive Gender. The phenomenological perspective was based on a humanistic. In addition, there was the qualitative methodology.

The group therapy was done with the afore-mentioned views and oriented to explore the central axis of the areas that were affected by intra-familial violence, to express feelings, to produce behavior and cognitive changes.

The methods that were used in the support group were that everyone pays attention to the behaviors, the ideas, and the expressions of each woman a thing that stimulated the oriented group to encourage the women in therapy to be the ones who recognized the tools to confront the violence with which they lived.

Key words: Intra-familial violence, therapeutic group, Infirmary intervention 


\section{INTRODUCCION}

Las diversas manifestaciones de la violencia intrafamiliar, se ensaña contra el cuerpo de las personas del grupo familiar percibidas como más débiles y dependientes. Pero, este cuerpo no es solo físico, es un cuerpo psíquico y social que ve afectada su identidad en aspectos tales como: integridad, autoimagen, valor, patrimonio, aspiraciones, reconocimiento, sexualidad, relaciones interpersonales y salud (Baró, 1988). Este tipo de violencia es uno de los muchos problemas que envuelven a nuestra sociedad. y es evidente su seriedad en todos los sectores socioeconómicos, religiosos, culturales, étnicos y en todas las regiones del país. Por tal razón, debe ser tratado como un problema social y no como un asunto privado. Por esto, la violencia intrafamiliar ha cobrado gran importancia e interés como lo confirma la Organización de los Estados Americanos (OEA) cuando aprobó en Belem do Pará, Brasil, una convención para prevenir, sancionar y erradicar la violencia contra la mujer en el continente. Dicha convención establece el derecho de toda mujer a "una vida libre de violencia, tanto en el ámbito público como en el privado”. (La Nación, 1994)

En 1995, Costa Rica firmó y ratificó dicha convención, la cual reconoce que la violencia contra las mujeres constituye una violación de los derechos humanos y exige, el respeto irrestricto de todos los derechos de las mujeres, como condición indispensable para su desarrollo individual, social, así como su plena participación en todas las esferas de la vida. El Estado costarricense además aprobó sin reservas la Declaración y Plataforma de Acción de Beijing, que establece expresamente que los Gobiernos deben formular y 
aplicar a todos los niveles apropiados, planes de acción para la erradicación de la violencia contra las mujeres.

En el ámbito nacional, en marzo de 1996 fue aprobada la Ley contra la Violencia Doméstica (Ley No. 7586), que regula la aplicación de las medidas de protección necesarias para garantizar la vida, la integridad, la dignidad y los derechos patrimoniales de las personas afectadas por la violencia intrafamiliar. Además, esta ley otorga al Instituto Nacional de las Mujeres la responsabilidad de ser el ente rector de las políticas públicas en materia de detección, atención y prevención de la violencia intrafamiliar, una de las manifestaciones más epidémicas y destructivas de la violencia de género. A pesar de que la Ley contra la Violencia Doméstica ha resultado una herramienta fundamental para la protección de las mujeres, en muchas ocasiones no ha resultado suficiente, porque al no ser de naturaleza penal, no implica sanciones. Por tanto, la violencia contra las mujeres sigue quedando impune, debido a que no se contemplan delitos específicos en el Código Penal. Por esta razón, se ha presentado un proyecto de ley a la Asamblea Legislativa, que busca penalizar la violencia en contra de las mujeres. Este proyecto se encuentra en proceso de discusión en el momento actual.

En estadísticas recientes queda demostrado cómo la problemática de la violencia intrafamiliar en Costa Rica está aumentando. De acuerdo con cifras suministradas por el Departamento de Planificación, Sección de Estadísticas del Poder Judicial, en 1997 se recibieron 15.396 denuncias por violencia intrafamiliar, un año después la cantidad ascendió a 20.996 y en 1999 la cifra de denuncias fue de 26.437 (La Nación, 2000). A las estadísticas anteriores se deben sumar las del año 2000, que indican que para ese año 25 mujeres fueron asesinadas, hechos que evidencian contundentemente la problemática de 
la agresión contra las mujeres. (INAMU, 2001). En Costa Rica, la violencia contra las mujeres ha cobrado en esta década, promedialmente, la vida de dos mujeres cada mes, asesinadas a manos de conocidos y desconocidos.

Queda demostrado que la violencia contra las mujeres es un hecho que tiene una gran incidencia en Costa Rica y que además, no se presenta como una situación aislada para las mujeres. Más bien, es un hecho que le ocurre frecuentemente a cada una trayendo consigo un sinnúmero de secuelas físicas y psicológicas. (Ferreira, B. Graciela. 1995)

Por todo esto, se hace necesaria la intervención profesional de la enfermera/o, la cual gracias a los conocimientos adquiridos durante su proceso de enseñanza, tiene la capacidad de identificar y valorar las consecuencias psicosomáticas y físicas que provoca este tipo de violencia, además de implementar acciones de intervención en la atención de las necesidades las mujeres sobrevivientes de violencia intrafamiliar. Específicamente la enfermería en Salud Mental, según refiere Cook (1993: p.74), está dirigida a tener un impacto tanto a nivel preventivo como correctivo, sobre los problemas emocionales y sus secuelas, a la vez se preocupa de la promoción de una óptima salud mental para la sociedad.

Considerando lo anteriormente mencionado, fue de mucha importancia desarrollar un grupo terapéutico de apoyo dirigido hacia las necesidades de las mujeres sobrevivientes de violencia intrafamiliar, con el fin de fomentar el reconocimiento de sus capacidades para modificar y resolver situaciones de la vida cotidiana, además de mejorar en cada una de las participantes su salud mental. 


\section{SUJETOS Y MÉTODOS}

El desarrollo del proceso terapéutico, se llevó a cabo en la Clínica de la Caja Costarricense de Seguro Social Dr. Ricardo Jiménez Nuñez, ubicada en el cantón de Goicoechea, catalogada como una clínica de atención integral tipo 4 con una población mayor de 50.000 habitantes, durante los meses de octubre, noviembre y diciembre del 2001. En total las sesiones fueron diez, realizadas los viernes, en un período de tres horas cada una.

El grupo estuvo conformado por 16 mujeres entre las edades de 19 a 70 años, anteriormente valoradas y referidas por la psiquiatra de la institución, por motivos tales como: depresión reactiva, depresión mayor recurrente, llanto frecuente, depresión severa, estrés post traumático por violencia intrafamiliar, sobrevivientes de violencia sexual e intento de autoeliminación.

Se procedió a contactarlas con el fin de tener el primer encuentro para motivar su participación en el proceso grupal, además de explicarles los contenido a desarrollar.

Para la selección de las participantes se tomó en cuenta los siguientes criterios: Que cumplieran con la mayoría de edad, no presentaran patologías psiquiátricas asociadas, que estuvieran vivenciando la problemática de la violencia intrafamiliar, residir cerca de la clínica como un factor que pudiera facilitar y asegurar la asistencia y comprometerse a asistir a la totalidad de las sesiones en el horario establecido.

Metodologia utilizada. El presente trabajo consistió en una Práctica dirigida de Graduación, la cual le da a la facilitadora la posibilidad de ser creativa e innovadora, al aplicar el conocimiento teórico - metodológico adquirido durante el proceso de 
enseñanza, que en este caso en particular, es la ciencia de la enfermería. (Brenes. 2000: p.12)

Para su desarrollo, se utilizó la perspectiva fenomenológica desde el enfoque género sensitivo. Esta perspectiva hace énfasis en las experiencias subjetivas de la persona, tomando en cuenta sus ideas, sus vivencias y sus sentimientos, lo anterior captado desde el marco de referencia interno del ser humano/a que las experimenta. El modelo de intervención género sensitivo, sostiene que todas las personas han sido afectadas adversamente por la estructura socio-cultural sexista. Examina las contradicciones inherentes al intento individual para crecer, mientras se vive en un ambiente social que enseña los usos inapropiados del poder y limita el acceso a los recursos, basándose en el género. El eje central de la intervención es el empoderamiento. Busca que la persona afectada revise sus concepciones acerca de la identidad femenina y masculina, reconozca las diferentes formas de presión y desarrolle estrategias para lograr su independencia y autonomía. (Ramellini. 1997: p.45).

Con el fin de respaldar la perspectiva fenomenológica, se hizo uso del enfoque humanístico el cual considera al ser humano/a como un todo indivisible que debe ser estudiado/a integralmente, en donde la persona busca su expansión y su plenitud en el proceso vital. Este enfoque fue de gran ayuda, para potenciar el cambio en las mujeres y para fundamentar la importancia del protagonismo de las mismas en dicho proceso. A lo anterior se debe sumar el uso de la metodología cualitativa, ya que aborda a la persona desde un punto de vista integral, considerándolo/a como un todo y no reducido a variables.(Taylor. y Bogdan. 1986: p.54). 
El proceso grupal se hizo acorde con los enfoques anteriormente mencionados, ya que buscó primordialmente el protagonismo de las miembras del grupo para lograr el reconocimiento de sus estrategias de enfrentamiento, que aplican en las situaciones de la vida cotidiana.

Para aprehender la realidad de la población y conocer las estrategias de enfrentamiento que utilizan las mujeres afectadas por violencia intrafamiliar en la vida cotidiana, se utilizó al iniciar el proceso grupal la técnica de Grupo Focal.

Para crear el proceso grupal con las participantes y de acuerdo a su realidad, se consideró la información recopilada al realizar la técnica de grupo focal para la selección de los temas a ser tratados durante el proceso terapéutico.

Para buscar el protagonismo de las mujeres que están participando en el reconocimiento de sus capacidades para modificar y resolver situaciones de la vida cotidiana, se tomó en cuenta las características fundamentales de la metodología participativa, con el fin de potenciar la criticidad que promueva una práctica consciente y transformadora de la realidad en que están inmersas.

En relación con el análisis y la evaluación del proceso grupal, se realizó un análisis de la información obtenida en el cuestionario de entrada, así como de los temas desarrollados en cada una de las sesiones, además, se hizo la evaluación y el análisis de los objetivos citados en cada una de las sesiones, basándose en los logros alcanzados por las participantes, así como la evaluación participativa permanente durante el desarrollo de las terapias grupales. Al finalizar el proceso, se utilizó nuevamente la técnica de grupo focal para evaluar los resultados en relación con los objetivos planteados. 


\section{RESULTADOS}

Considerando lo mencionado por Barrantes (1999: p.16), el análisis de los datos se llevó a cabo a través de una descripción densa, cuyos rasgos característicos son, que es interpretativa y que lo que interpreta es el flujo del discurso social.

Analisis de la información recopilada en el cuestionario de entrada: De acuerdo a las edades es importante notar la heterogeneidad en las participantes del grupo, esto indica que la violencia intrafamiliar no respeta edades. Hecho que confirma, que esta problemática se debe más por la condición genérica, ya que la construcción de la identidad femenina en una sociedad patriarcal, vulnerabiliza a la mujer a vivir en una posición inferior con respecto al hombre. De igual manera, el nivel académico y socioeconómico, no inciden en la presencia o no de violencia contra las mujeres, como erróneamente se cree. Lo anterior se ve representado en el grupo, porque las participantes cuentan con diferentes niveles académicos, que van desde la primaria incompleta hasta el grado de educación superior completa. Del mismo modo, el nivel socioeconómico de las participantes es variado, lo que demuestra que la problemática de la violencia intrafamiliar no es exclusiva de la clase socioeconómica baja, como se ha estigmatizado a través del tiempo. Con respecto al estado civil de las participantes, se observó que la mayor parte de ellas viven con su compañero en condición de casada o en unión libre. Esto representó un hecho importante, ya que algunas de las mujeres que viven con su pareja, sufren de situaciones de violencia más continuas y severas, que las que viven separadas. Otras de las características de las mujeres participantes, es que todas ellas tienen uno o más hijos/as. Esta situación posee gran relevancia, ya que la presencia de hijos dentro del hogar, es una de las razones que comúnmente verbalizan las mujeres para 
no separarse de los agresores, al referir que los hijos/as necesitan de un padre. La condición de salud manifestada por las mujeres participantes en el grupo, denota un deterioro físico y psicológico. Todas refirieron tener como diagnóstico médico la depresión, la cual se relaciona directamente con la situación de violencia intrafamiliar que viven. A raíz de esto, también presentan otras enfermedades que igualmente se relacionan con esta problemática, tal es el caso, de los trastornos cardiovasculares, gastrointestinales, dolores musculares y cefaleas recurrentes. De esta manera, se observó la interrelación entre sus vivencias de violencia y las respuestas orgánicas, que generan un círculo vicioso de síntomas físicos y psicológicos. Por esta razón, la mayor parte de las participantes toman medicamentos, entre los que se encuentran: antidepresivos, ansiolíticos, antiinflamatorios y antihipertensivos. Haciendo referencia a los tipos de violencia que han experimentado las mujeres, se puede rescatar que todas han vivido violencia emocional y en menor medida los otros tipos de violencia. Lo anterior demuestra como las miembras del grupo, han vivido por parte de sus parejas un constante abuso del poder a través de, acciones u omisiones que dañan su integridad física y psicológica. Este desequilibrio de poder, es constante y se perpetúa a lo largo de la relación, esto hace que se mantenga un ciclo de violencia que le dificulta a la mujer salir de esta situación de agresión. En relación con la participación previa en un grupo terapéutico, de las dieciséis integrantes del grupo, diez verbalizaron no haber asistido a uno anteriormente. Lo anterior se puede relacionar con varios factores, como la escasez de grupos de apoyo que brinda la clínica Dr. Ricardo Jiménez Nuñez, la falta de información que tienen las mujeres sobre los diferentes lugares que brindan apoyo, además, se debe considerar el temor y el dolor emocional que representa reconocer ante un grupo de iguales que se 
sufre de violencia intrafamiliar; esto provoca que muchas mujeres, se rehúsen a participar en un proceso terapéutico. Sin embargo, las miembras del grupo que sí habían participado en un proceso terapéutico previo, tuvieron un comportamiento muy participativo. Este hecho aceleró la etapa de incertidumbre y ayudó a fomentar la cohesión grupal entre las mujeres.

\section{A continuación se presenta los logros alcanzados por las participantes según la evaluación de los objetivos planteados en cada una de las sesiones:}

- Por medio de la discusión de preguntas abiertas (grupo focal), las participantes expresaron las estrategias de enfrentamiento que utilizan en las situaciones difíciles de la vida cotidiana, que les permite seguir adelante: Buscar ayuda espiritual y profesional, hacer valer sus derechos, mantener la calma y evitar incidentes agudos.

- A través de una lluvia de ideas se propusieron las normas del grupo, donde se expusieron las siguientes: Confidencialidad, apoyo, puntualidad, respeto, positivismo, espacio para hablar, no restar valor a la situación de mi compañera, expresar lo que sentimos, brindar espacio y oportunidad para todas hablar, constancia, no sentirse obligada a participar, valorar a mis compañeras

- Al realizar la actividad "Lo que me gustaría lograr", las participantes manifestaron lo siguiente: Deseos de salud emocional y de una mejor salud física, necesidad de superar su situación de violencia, respeto y autosuperación.

- Los comentarios por parte de las participantes, en relación a los conocimientos adquiridos fueron: Que las mujeres no nacen como seres inferiores a los hombres, más bien, es la construcción de la identidad femenina la que provoca esto. 
- Reconocieron estrategias que las pueden ayudar a superar la situación de violencia en la que viven, tales como: autorespeto, empoderamiento, validar los propios derechos, buscar la igualdad tanto dentro del hogar como fuera de éste, tomar decisiones, pensar en ellas mismas como seres humanas independientes y fuertes, ponerse en un primer plano con respecto a sus familias y poner en práctica límites claros en sus relaciones con los/las demás.

- Comprendieron mejor la problemática de la violencia intrafamiliar y sus manifestaciones, lo que permitió que las mujeres elaboraran acciones y estrategias que deben de poner en práctica, para vivir su cotidianidad lo mejor posible.

- Identificaron los diferentes tipos de violencia y sus repercusiones.

- Aprendieron sobre el uso, efectos y alcance de los medicamentos prescritos por la psiquiatra.

- Identificaron diferentes soluciones que pueden utilizar para procurarse un lugar seguro dentro de su familia y vivir una vida libre de violencia.

- Reconocieron que la culpa es un factor determinante para que las mujeres actúen en beneficio de los demás dejando de lado el propio ser, ya que manipula desfavorablemente la voluntad de las mujeres.

- Conocieron las diferentes redes de apoyo que les brindan ayuda y contención.

- Lo más importante, es que reconocieron sus fortalezas y valores como personas.

- Durante el desarrollo de las sesiones, se llegó a establecer un proceso en donde consiguieron alcanzar el propósito principal: fomentar en las mujeres sobrevivientes de VIF, el reconocimiento de sus capacidades, para modificar y resolver situaciones de la vida cotidiana. 


\section{DISCUSIÓN Y COMENTARIOS}

1) La terapia grupal de apoyo se basó en la perspectiva fenomenológica desde el enfoque género sensitivo, la cual ve los hechos desde el punto de vista individual y según las situaciones que vive cada ser humano/a

2) Las diferencias existentes entre los géneros, en aspectos tales como el cultural, el físico y el social, son el punto de partida para la inferiorización del sexo femenino; tanto en el ámbito privado como en el público

3) De acuerdo a las vivencias de las miembras del grupo, la violencia intrafamiliar se puede manifestar de diferentes formas: física, psicológica, patrimonial y sexual. Estos abusos son recibidos por parte de sus parejas, quienes gozan de mayor poder. Esta situación hace que la relación de pareja se transforme en un constante atropello a la dignidad de la mujer la cual se ve humillada, lesionada y desvalorizada.

4) La dinámica de la violencia intrafamiliar, resulta sumamente dañina para la personalidad de la mujer la cual se ve sometida a constantes abusos. Por lo tanto, es inevitable que el autoestima de quien la vive se vaya desgastando poco a poco, esto en gran medida dificulta la ruptura de la violencia, ya que la mujer encauza todas sus energías a construir estrategias de sobrevivencia.

5) Se debe de considerar a las mujeres sobrevivientes como mujeres fuertes, no solo porque han sobrevivido al maltrato, sino porque en el camino siguen realizando las tareas cotidianas a pesar del desgaste emocional y físico que conlleva la agresión.

6) Es importante dejar de lado las frases y pensamientos que estereotipen a la mujer sobreviviente de violencia, ya que estas concepciones inducen a las mujeres a mirarse impotentes y llenas de limitaciones, pero sí por el contrario se les considera como seres 
adultas con derechos y deberes, en igualdad de condiciones que cualquier otra persona, capaces de asumir la responsabilidad de su vida y de construirse un futuro exitoso, se les estará estimulando para que tomen decisiones, crean en sus capacidades y disminuyan sus temores y así logren enfrentar con mayor libertad y fortaleza la situación de agresión. 7) A pesar de los múltiples efectos de la violencia intrafamiliar, muchas mujeres han podido ser capaces de salir de la agresión. La única respuesta posible es que en las mujeres hay una gran fortaleza y vitalidad que no muere con el maltrato, por lo tanto no se debe de creer que se les debe marcar el camino o llevarlas de la mano para resolverles sus problemas.

8) Es indiscutible que los efectos de la violencia intrafamiliar tienen consecuencias muy negativas a nivel fisiológico, ya que el estilo de vida que llevan las mujeres sobrevivientes, ocasiona un fuerte desgaste en su salud.

9) Se debe rescatar la importancia que juega el ciclo vital de las mujeres participantes en el grupo ya que según éste, podemos ver las distintas etapas en las que se encuentran y por consiguiente las tareas que cumplen y dejan de cumplir, siendo esto último el más importante, porque desencadena situaciones criticas cuyo origen son las situaciones de violencia intrafamiliar. Algunos ejemplos de las tareas que no se están cumpliendo son las siguientes: aprender a vivir con el compañero, consolidar las relaciones con el cónyuge y disfrutar de comodidad emocional.

10) El impacto de la violencia intrafamiliar dificulta que las mujeres reconozcan sus propios recursos para enfrentarla, ya que ésta provoca en ellas percepciones negativas sobre sus capacidades para salir adelante. Sin embargo el apoyo entre iguales y la adecuada facilitación terapéutica que se da en los procesos grupales, ayudan a destruir 
las expectativas negativas que las mujeres tienen acerca de su futuro, dando paso a un nuevo aprendizaje que las lleva recocer su potencial como seres humanas.

11) Las mujeres sobrevivientes de violencia intrafamiliar generalmente no son consientes de sus capacidades y de las estrategias que utilizan diariamente para enfrentar dicha problemática, razón por la cual el proceso terapéutico resultó ser de gran valor para que ellas tomaran conciencia de sus propios recursos y al mismo tiempo, lograran compartir y aprender de las demás compañeras.

12) Es una responsabilidad de todas y todos los profesionales de salud mental conocer las repercusiones que para las mujeres tienen el uso de los enfoques que las victimizan, las etiquetas de enferma mental o loca, que se asocian con cualquier persona en tratamiento o terapia, ya que son instrumentos poderosamente revictimizantes. El uso de ese tipo de términos descalificadores ha sido uno de los mecanismos ampliamente empleados para garantizar el control social y la discriminación de género, por esto el profesional que tenga contacto con estas personas esta encargado de eliminar cualquier concepto que las inferiorice.

13) Al concluir con el proceso terapéutico se demostró la importancia y la necesidad de la enfermera de salud mental dentro del equipo multidiciplinario de salud, dado que su formación integral le permite abarcar tanto las áreas psicofisicas como las psicoafectivas de las personas que se han visto o están siendo afectadas en estos campos.

14) Para nosotras como facilitadoras, la experiencia adquirida durante el desarrollo de este trabajo fue satisfactoria a nivel personal y profesional, ya que en el primero nos facilitó hacer rupturas de nuestros propios mitos acerca de la construcción identitaria femenina, de la violencia hacia las mujeres, de la violencia intrafamiliar y la dinámica 
alrededor de ésta. En relación al campo profesional nos permitió desarrollar nuestra capacidad como facilitadoras dentro de un proceso terapéutico, a esto le sumamos los grandes conocimientos adquiridos en relación a la temática de la violencia intrafamiliar. Recomendaciones. Ampliación y diversificación de los servicios de la Caja Costarricense del Seguro Social, para la atención de las mujeres afectadas de violencia intrafamiliar. Desarrollar un programa continuo de sensibilización y educación para el personal que labora en instituciones que atienden a mujeres que vivencian la problemática de violencia intrafamiliar. Desarrollar grupos terapéuticos guiados por la enfermera de salud mental en los diferentes centros de salud, para tratar la problemática de la violencia intrafamiliar y grupos de autoayuda en los centros de salud, para que las mujeres cuenten con apoyo y contención, con el fin de enfrentar las situaciones difíciles causadas por la violencia intrafamiliar. Debido al impacto de la violencia intrafamiliar en los hijos e hijas de las mujeres sobrevivientes de violencia, se recomienda terapias de apoyo que traten las secuelas de la VIF. Se hace necesario que el personal de las instituciones que atiende a las mujeres sobrevivientes de VIF, conozca las diferentes redes de apoyo con las que puede contar esta población. Se insta a los profesionales de enfermería en Salud Mental, a ampliar sus conocimientos sobre el tema de la violencia intrafamiliar o la violencia de género, con Instituciones como el INAMU (Instituto Nacional de la Mujeres) ó CEFEMINA (Centro Feminista de Información y Acción), con el fin de brindar una mejor atención a las mujeres afectadas por la violencia intrafamiliar.

Facilidades. Anuencia por parte de la psiquiatra de la clínica Dra. Guisell Brenes y de la coordinadora del área de Salud Mental Dra. Hellen Ulloa Magg para desarrollar el proceso terapéutico. La Dra. Guiselle Brenes nos facilitó los nombres de las mujeres que 
conformaron la población del grupo terapéutico. Se nos brindó un espacio físico adecuado para el desarrollo de las sesiones. Contamos con una amplia participación y asistencia de las mujeres que conformaron el grupo, lo que contribuyó al adecuado desarrollo del mismo.

\section{BIBLIOGRAFIA}

Alvarez, Rodrigo. (1990). Guía Práctica para la Docencia en Salud. San José: Editorial P.A.S.C.AP.

Barrantes E. Rodrigo. (1999). Investigación Un Camino al Conocimiento. San José: EUNED

Baró, Martín. (1998) Revista Costarricense de Psicología. San José. Número 12 -13. Dic.

Basaglia, Franca. (1985). Mujer Locura y Sociedad. México, D.F: Editorial de la Universidad Autónoma de México. D.F.

Batres. G. Claramunt. Cecilia. (1990). La violencia contra la mujer en la familia costarricense: un problema de salud pública. San José: Fundación Ser y Crecer.

Calvo, Yadira. (1994). "La Bella y la Bestia”. La República. (San José), 31 de mayo.

Claramunt, Cecilia. (1998). Casitas Quebradas: El problema de la violencia doméstica en Costa Rica. San José: EUNED.

Corsi, Jorge. (1994). Violencia Familiar. Buenos Aires: Editorial Paidós.

Cook, Sue. (1993). Fundamentos Esenciales de Enfermería en Salud Mental. Barcelona: Editorial Interamericana.

Courtois, Christine. (1988). Healing the Incest Wound. U.S.A. W.W. Norton \& Company.

Delgado, Juan M. Gutiérrez, Juan. (1999). Métodos y Técnicas Cualitativas de Investigación en Ciencias Sociales. Madrid: Editorial Síntesis. S.A.

Facio. M. Alda. (1993). El Derecho Patriarcal Androcéntrico. San José: Fundación Ser y Crecer.

Ferreira, Graciela B. (1995). Hombres violentos, Mujeres Maltratadas. Buenos Aires: Editorial Sudamericana.

Fundación Ser y Crecer. 1993. Violencia contra la mujer. San José.

Fundación Ser y Crecer. 1995. Violencia Física Contra la Mujer. San José.

Fundación Ser y Crecer. 1990. Amor aterrorizante. San José.

Fundación Ser y Crecer. (1993). Taller introductorio "Orígenes de la Violencia Sexual y Física contra las Mujeres”. San José.

Instituto Nacional de las Mujeres. (1999). Sentir, pensar y enfrentar la violencia intrafamiliar. Lecturas complementarias. San José: Editorial Grafos. S.A.

Instituto Nacional de las Mujeres. (2001). Lista de Mujeres Asesinadas en el 2000.

Loaiza, N. Vanessa. (2000). La Nación. (San José), 8 de enero.

La Nación (1994). ¡No más golpes!. (San José), 20 de junio, VIVA / 4. 
Lagarde, Marcela. (1992). Identidad de Género. Memoria del curso del centro OLOF. Palme.

Lagarde, Marcela. (1994).Compendio de Documentos Género y Derechos Humanos. San José: IDH.

Lordoño, Argelia. (1992). Hacia la Recuperación del Asombro: La Violencia Contra la Mujer Asunto de Salud Pública. En Memoria Seminario Subregional sobre Violencia contra la Mujer. Managua: OPS.

Mizrahi, Liliana. (1990). Las Mujeres y la Culpa. Buenos Aires: Grupo Editor Latinoamericano.

Mora Carpio, Lucila. (1992). Violencia doméstica y construcción de identidad femenina.

Naciones Unidas, ILANUD. (1991). Programa piloto permanente en el tema de la violencia dirigido a técnicos de Ministerio de Justicia. San José.

Pastor, Rocío. (1994). "Mujeres Rompen el Silencio". La Nación. (San José), 13 de marzo.

Peplau, E. Hildegard. (1990). Relaciones Interpersonales en Enfermería. Barcelona: Editorial Salvat.

Pérez, N. Dellanira. (1995). Autoestima. Centro de nuestra vida. San José: Editorial Colectivo de Mujeres Pancha Carrasco.

Pineda. B. Elia. (1990). Metodología de la Investigación. Manual para el desarrollo del personal de salud. Editorial OPS.

Quirós Rodríguez, Edda. 1999. “... y no viví feliz para siempre...”. San José: Editorial Grafos S.A.

Ramellini Centella, Teresita. Mesa Peluffo Sylvia. (1997). Estrategias de intervención especializada con personas afectadas por la violencia intrafamiliar. Emprendiendo un camino. San José: Editorial Grafos S.A.

Shrader, Elizabeth. Sargot, Moserrat. (1998). La ruta crítica que siguen las mujeres afectadas por la violencia intrafamiliar. Washington, D.C.: Editorial Organización Panamericana de la Salud.

Sue, Cook. (1993). Fundamentos esenciales de enfermería en Salud Mental. Barcelona: Editorial Mc Graw - Hill.

Taylor S. y Bogdan R. 1986. Introducción a los métodos cualitativos de Investigación. Buenos Aires: Editorial Paidós.

Walker, Lenore. (1979). Las Mujeres agredidas. Nueva York: Harper and Row Publishers, INC.

Walker, Lenore. (1991). Temas sobre Violencia Contra las Mujeres. Proyecto de Capacitación Dirigido a Técnicos del Ministerio de Justicia. San José: ILANUD.

Walker, Lenore. (1990). Terrifying Love: Why battered woman Killand how society responds. Nueva York. Harper and Row Publishers, INC.

Walker, Lenore. (1980).The Bettered Woman. Nueva York. Harper and Row Publishers, INC. 